\title{
A morphological classification of sperm defects in the ostrich (Struthio camelus)
}

\author{
Lizette du Plessis $^{1 *}$, Irek Malecki ${ }^{2,3}$, Maud Bonato ${ }^{2}$, Marna Smith $^{2}$, Schalk Cloete ${ }^{4}$, \\ John Soley ${ }^{5}$
}

${ }^{I}$ Electron Microscope Unit, Department of Anatomy and Physiology, Faculty of Veterinary Science,

University of Pretoria, Onderstepoort, South Africa

${ }^{2}$ Department of Animal Science, University of Stellenbosch, Matieland, South Africa

${ }^{3}$ School of Animal Biology, Faculty of Natural and Agricultural Sciences, University of Western Australia,

Crawley, Australia

${ }^{4}$ Directorate Animal Sciences, Elsenburg, South Africa

${ }^{5}$ Department of Anatomy and Physiology, Faculty of Veterinary Science, University of Pretoria,

Onderstepoort, South Africa

*Corresponding author: e-mail: lizette.duplessis@up.ac.za; Telephone: +27 125298212 ;

Postal address: Faculty of Veterinary Science, University of Pretoria, Private Bag X04, Onderstepoort 0110, South Africa 


\section{ABSTRACT}

Commercial ostrich farming is constrained by the absence of a formal animal recording and evaluation scheme as well as by current farming practices. Artificial insemination may have an important role in overcoming these limitations, but requires a thorough knowledge of sperm morphology. Although the morphological characteristics of normal ostrich sperm have been documented, little information is available on the incidence and structural peculiarities of defective sperm in this species. Semen smears were prepared from the ejaculates of five ostriches (Struthio camelus), stained and evaluated. Defects were observed in $17 \%$ of sperm studied. Tail defects constituted the most common anomaly. Various forms of bending were the main tail defect, ranging from gentle to acute bends of the principal piece, Dag-like coiling at the head base, sharp reflexes of the midpiece as well as coiling of the endpiece. In contrast, head defects were comparatively low in frequency, with macrocephalic sperm being the defect most frequently observed in this region. Bent, microcephalic, acephalic and round sperm heads were also noted but were few in number. Cytoplasmic droplets occurred frequently in the fixed smears, either associated with the sperm or as free-lying droplets. A small percentage of sperm with multiple defects was recorded. The incidence of morphologically normal sperm in ostrich semen compared favorably with that reported in emu semen, another commercially farmed ratite. However, the range of defects differed appreciably between the two species. Sperm tail anomalies were the most frequent category in the ostrich, whereas head defects comprised the main grouping in the emu.

Keywords: Ostrich, Light microscopy, Sperm defects, Sperm morphology 


\section{Introduction}

The commercial ostrich industry is characterized by a complete absence of a performance recording and evaluation scheme, as is common in other livestock (Cloete et al., 2008). Commercial ostrich farming therefore relies on natural reproduction for the supply of slaughter birds. Commercial breeder birds are often kept in colonies that may exceed 100 birds at a male:female ratio of 5 to 6:10 (Lambrechts et al., 2004). This excess of male birds for the sole purpose of breeding adversely affects the economic viability of the industry. Additionally, this system makes it difficult to efficiently improve the genetic quality of farmed ostriches in the industry (Bonato et al., 2011; Cloete et al., 2008; Kawka et al., 2007). It has been suggested that the use of artificial insemination (AI) could address these limitations (Bonato et al., 2011; Cloete et al., 2008; Malecki et al., 2008). However, the routine availability of high quality semen is a prerequisite for an AI program to be successful (Bonato et al., 2011; Malecki et al., 2008) and a number of studies have provided important information relevant to the ostrich spermiogram. These include the effects of the collection method, age of the male, month of the season and collection frequency on the ejaculate (Bertschinger et al., 1992; Bonato et al., 2011; Hemberger et al., 2001; Rybnik et al., 2012). The optimal time for the collection of semen, between male variation in semen characteristics, the effect of dilution on sperm viability and the effect of temperature and $\mathrm{pH}$ on the motility and viability of ostrich sperm have also been studied (Bonato et al., 2010, 2012; Ciereszko et al., 2010).

Although the morphology of normal ostrich sperm has been described by light microscopy (Berens von Rautenfeld, 1977; Retzius, 1911; Soley, 1992; Soley and Groenewald, 1999) and studied extensively by electron microscopy (Baccetti et al., 1991; Jamieson, 2007; Soley, 1992, 1993; Soley and Roberts, 1994), little information is available on the incidence and nature of sperm defects in this species. Hemberger et al. 
(2001) simply note that the number of abnormal sperm ranges from $5 \%$ to $26 \%$, with an average value of $17 \%$, without giving an indication of the types of defects observed. Berschinger et al. (1992) reported that the most common major sperm defects were "midpiece reflexes and coiled tails (Dag defects), partial or complete aplasia of the mitochondrial sheath, persistent cytoplasmic droplets and abnormally shaped heads", while Irons et al. (1996) identified "midpiece reflexes and cytoplasmic droplets" as being the most common defects. No information regarding the incidence of the defects was provided in these studies. The comprehensive review on reproduction in non-domestic birds (Gee et al., 2004), although describing the basic structure of ostrich sperm, provides no specific information on defective cells in this species. The only defect addressed in the ostrich is that of cytoplasmic droplets where the incidence and morphology of these structures have been described (Soley and Els, 1993; Soley et al., 1996).

Considering the importance attached to sperm morphology as one of the salient factors in the determination of fertility in the male ostrich (Bertschinger et al., 1992; Soley et al., 1991), and in view of the future potential selection of stud males for AI programs, it is important that a detailed classification of sperm abnormalities be made available for this species. This study, therefore, aims to describe and classify sperm defects in the ostrich (Struthio camelus) and provide the relative incidence of the defects.

\section{Materials and methods}

\subsection{Animals and location}

The study was conducted on the Oudtshoorn Research farm of the Western Cape Department of Agriculture (GPS co-ordinates: -33.631718 S, 22.257137 E) in the Little Karoo region of South Africa. The climate at the site is arid, with an average annual precipitation of $360 \mathrm{~mm}$. The farm houses a population of male ostriches trained to 
ejaculate in an artificial cloaca, using the dummy female method (Malecki et al., 2008; Rybnik et al., 2007). The animals were used extensively in research on semen traits (Bonato et al., 2010, 2011, 2012). The birds were maintained in single paddocks with limited browse material. Birds were provided with feed ad libitum and potable drinking water. Semen samples were collected on three separate occasions from five sexually active South African black ostriches, ranging from 3 to 8 years of age, during July and August 2013. Ethical clearance for the project was obtained through the Western Cape Department of Agriculture, reference number DECRA R09/24.

\subsection{Microscopy}

The ejaculated semen samples were immediately fixed in $2.5 \%$ glutaraldehyde in 0.13M Millonig's phosphate buffer, pH 7.4 at an approximate ratio of 1:10. Smears were prepared from the fixed cell suspensions, air-dried for a minimum period of $24 \mathrm{~h}$ and stained with Wright's stain (Rapidiff ${ }^{\circledR}$, Clinical Sciences Diagnostics, Johannesburg, South Africa) in Coplin jars as previously described (Du Plessis and Soley, 2014).

One smear from each collection for each bird was examined with an Olympus BX63 light microscope (Olympus Corporation, Tokyo, Japan) using a 100x oil immersion objective (phase contrast illumination). The incidence of normal and defective sperm was determined for each bird by evaluating 300 cells per slide. Morphological defects were assessed and described according to the sperm region affected. Images of defective cells were digitally recorded using the Olympus cellSens Imaging Software (Olympus Corporation, Tokyo, Japan).

In addition, the linear dimensions (mean $\pm \mathrm{sd}$ ) of the head and tail of normal-sized sperm and macrocephalic cells, as well as the total length of the cells, were determined. A minimum of 25 normal cells from each bird and a total 26 macrocephalic cells, randomly 
selected between the birds, were measured. The measurements were processed using the Soft Imaging System iTEM software (Olympus, Münster, Germany).

\section{Results}

Normal ostrich sperm were typically filiform in appearance as previously described in the literature (Berens von Rautenfeld, 1977; Gee et al., 2004; Hemberger, 1996; Retzius, 1911; Soley 1992, Soley and Groenewald, 1999). The cells were $78.9 \pm 2.9 \mu \mathrm{m}$ long with the head (acrosome and nucleus) and the tail (midpiece, principal piece and endpiece) measuring $16.7 \pm 0.6 \mu \mathrm{m}$ and $62.2 \pm 2.5 \mu \mathrm{m}$, respectively. With phase contrast illumination the anteriorly tapered nucleus could clearly be distinguished from the rest of the cell by its luminescent appearance. The head was capped by a small pointed acrosome. The various parts of the tail were obvious by the staining intensity and differences in diameter. The slightly darker staining, approximately $3.0 \mu \mathrm{m}$ long midpiece represented the first part of the flagellum which continued as the long principal piece, terminating in the noticeably thinner and short endpiece (Fig. 1). The incidence of morphologically normal sperm was $83 \%$.

Defective ostrich sperm were divided into four broad categories as previously proposed for ratite sperm, namely, head defects, tail defects, multiple defects and cytoplasmic droplets (Du Plessis and Soley, 2011a). Head and tail defects were further defined according to the particular region of the cell affected (acrosome, nucleus, neck/midpiece, principal piece or endpiece). The incidence of the various defects, expressed as a percentage of total defects, is presented in Table 1.

Head defects comprised $9.2 \%$ of total sperm defects. These defects were exclusively restricted to the nucleus and no acrosomal abnormalities were observed. Head anomalies included macrocephalic (Fig. 2a), microcephalic (Fig. 2b) and acephalic (Fig. 2c) sperm as 


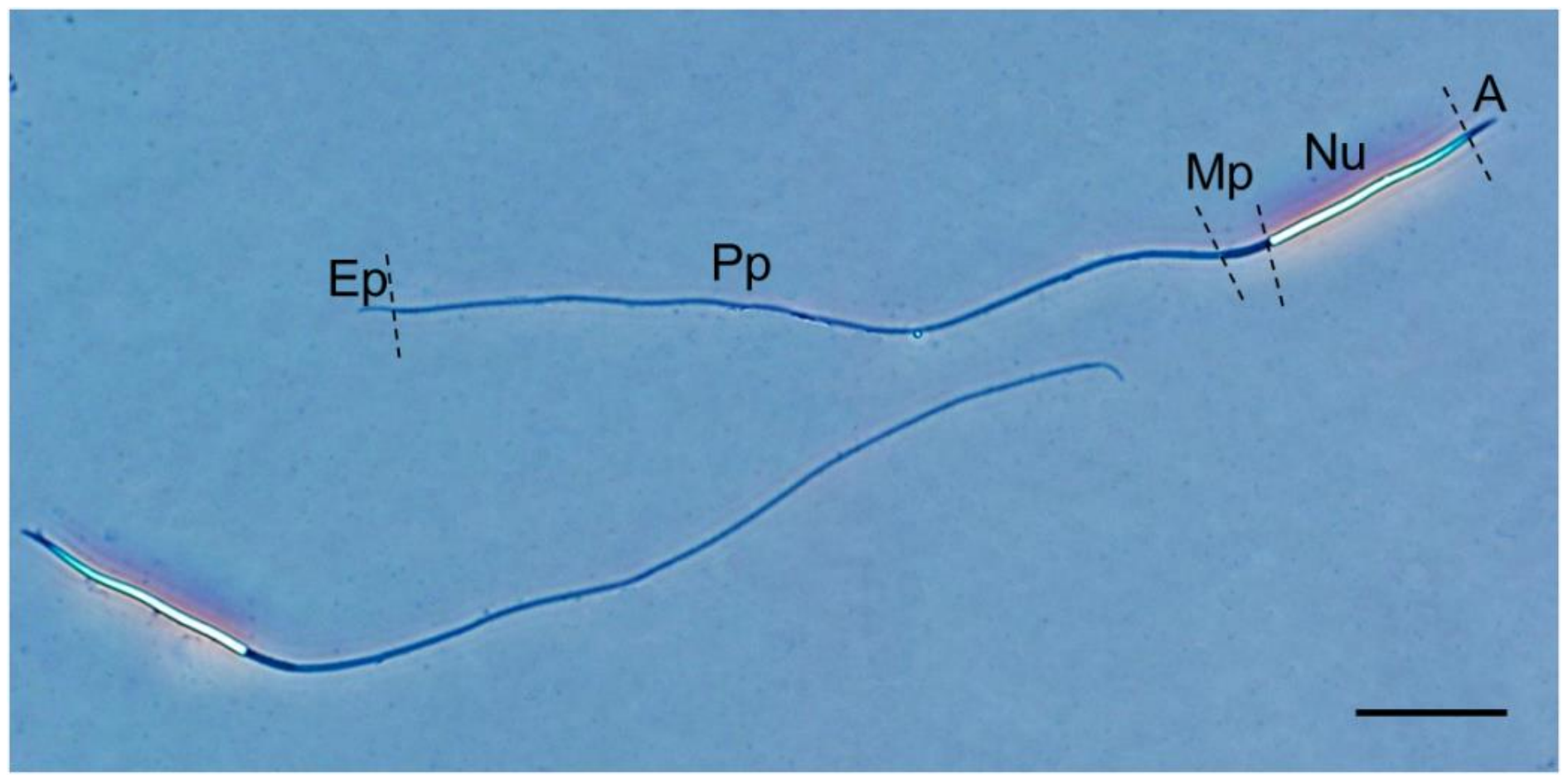

Fig. 1. Normal ostrich sperm. Wrights' stain, phase contrast illumination. A, acrosome; $\mathrm{Nu}$, nucleus; $\mathrm{Mp}$, midpiece; $\mathrm{Pp}$, principal piece; Ep, endpiece. Bar $=10 \mu \mathrm{m}$. 


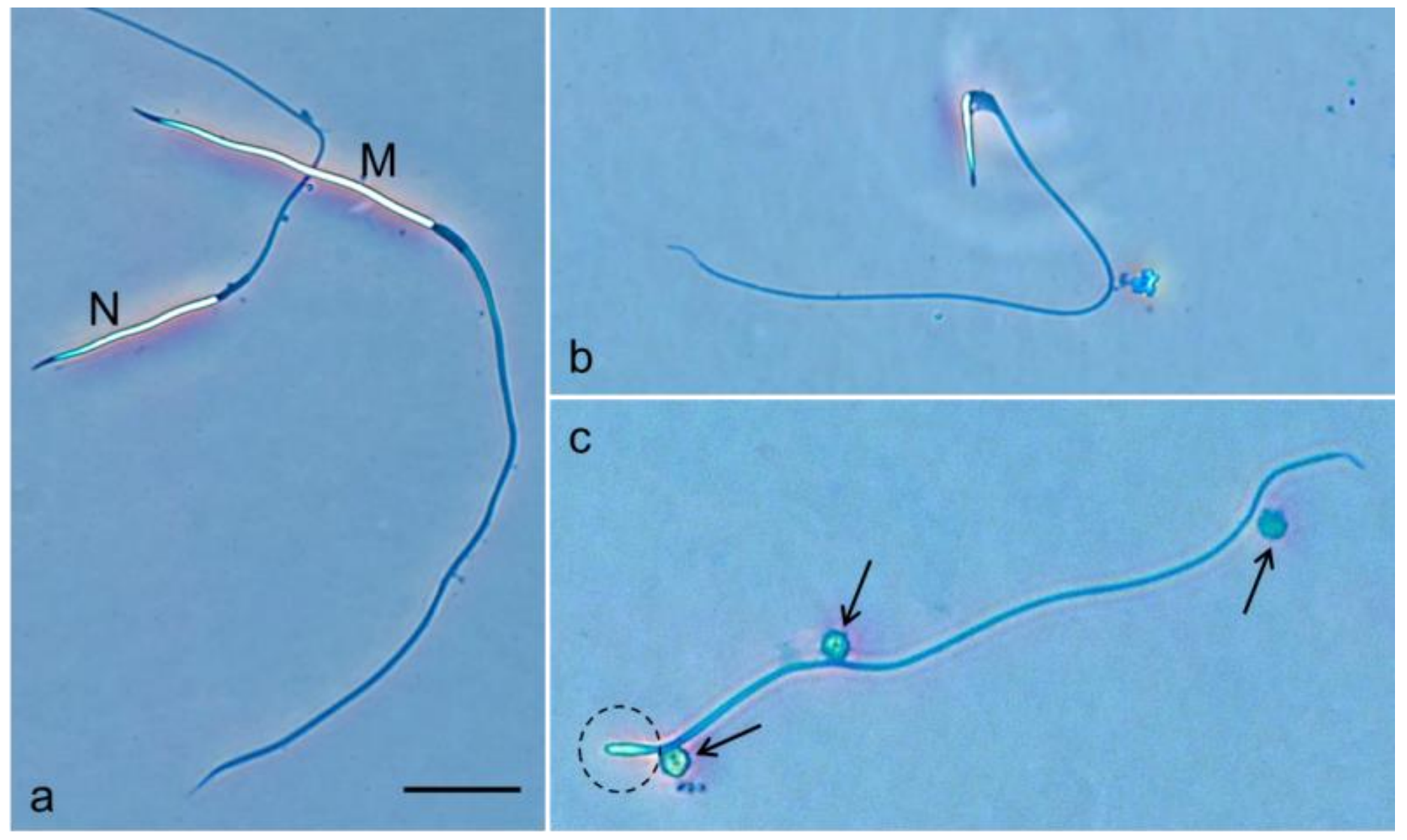

Fig. 2. Various forms of ostrich sperm head defects. (a) A macrocephalic sperm (M) with a visibly thicker tail. Note the length of the head compared to a normal sized sperm (N). (b) A microcephalic sperm with cytoplasmic material associated with the midpiece resulting in head/midpiece bending. Although not scored as such in the present study (see Table 1), this cell would represent a multiple defect. (c) Acephalic sperm (circled) with closely associated free cytoplasmic droplets (arrows). Bar $=10$ $\mu \mathrm{m}$. 


\section{Table1}

Classification and incidence of ostrich sperm defects determined from the evaluation of fixed and stained semen smears. The defects are expressed as a percentage of total sperm defects.

\begin{tabular}{lll}
\hline CATEGORY & REGION & SPECIFIC DEFECT \\
\hline Head defect & Nucleus & Bent $1.4 \%$ \\
$9.2 \%$ & $9.2 \%$ & Macrocephalic $7.7 \%$ \\
& & Microcephalic $<0.1 \%$ \\
& & Acephalic* \\
& & Round \\
\hline Tail defects & Neck/midpiece & Disjointed $8.4 \%$ \\
$72.1 \%$ & $19.8 \%$ & Abaxial $0.9 \%$ \\
& & Bent $10.5 \%$ \\
\cline { 2 - 3 } & Principal piece & Bent $44.3 \%$ \\
& $51.7 \%$ & Coiled $6.8 \%$ \\
& & Multiple $0.1 \%$ \\
& & Loose $0.5 \%$ \\
\cline { 2 - 3 } & Endpiece $0.6 \%$ & Coiling $0.6 \%$ \\
\hline
\end{tabular}

Cytoplasmic droplets

$17.5 \%$

Multiple defects

$1.2 \%$

*Observed when scanning the entire smear but not as part of the 300 cells counted when the incidence of the various defects was determined

well as head bending (Fig. 3) and deformed heads (Fig. 5a). The most commonly observed head defect (12.4\% of total defects) was macrocephalic sperm which displayed markedly longer heads $(26.5 \pm 1.6 \mu \mathrm{m})$ than those of normal sperm $(16.7 \pm 0.6 \mu \mathrm{m})$. No obvious difference was apparent in tail length between normal and macrocephalic sperm, reflecting a head:tail ratio of 1:3.7 for normal sperm and 1:2.3 for macrocephalic sperm.

Microcephalic sperm, which were rarely observed (Table 1), revealed typical head features (slender nucleus capped by a pointed acrosome) although these heads were $30 \%$ to $50 \%$ shorter than normal sized sperm heads. A few acephalic sperm (Table 1), characterized by the presence of a midpiece, principal piece end endpiece but devoid of a nucleus or 


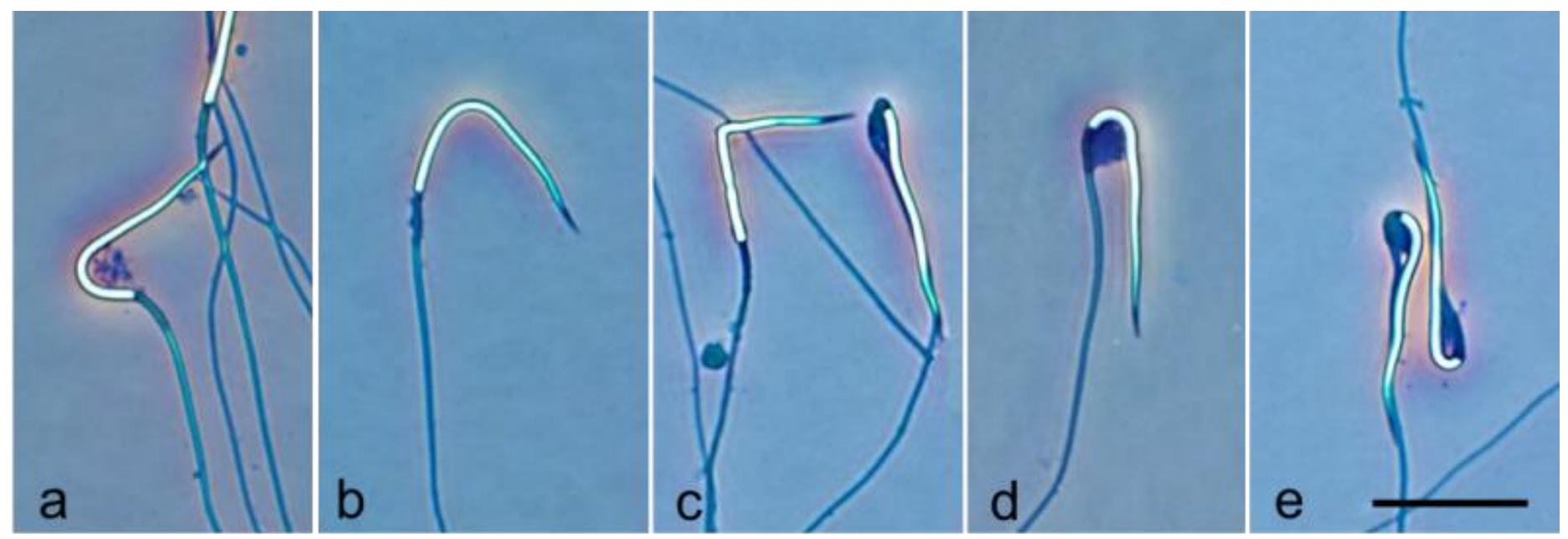

Fig. 3. Various forms of head bending noted in ostrich sperm, ranging from gentle bends $(\mathrm{a}, \mathrm{b})$ to more acute $\left(90^{\circ}\right.$ and $180^{\circ}$ ) forms $(\mathrm{c}-\mathrm{e})$. Note the presence of residual cytoplasmic material particularly in the more acute forms of bending, which often occurs in the vicinity of the nuclear base (d, e). Bar $=10 \mu \mathrm{m}$. 
acrosome, were noted (Fig. 2c). These cells were not considered to have loose tails as there was no evidence of loose or detached heads in the smears.

Bent heads formed $1.4 \%$ of total defects and were characterized by bending of the nucleus at any point along its length. The degree of bending varied from a gentle bend to more acute $90^{\circ}$ and $180^{\circ}$ bends (Fig. 3a-e). This defect was often associated with the presence of cytoplasmic material, particularly where bending occurred towards the base of the head (Fig. 3a, d, e). Exceptionally large cells characterized by a round or severely deformed head were occasionally observed (Fig. 5a). No distinctive acrosome or midpiece could be identified in these cells and the principal piece appeared thickened when compared to that of the other cells. These cells were classified as a multiple defect.

Tail defects formed the largest group of sperm anomalies observed in the ostrich, comprising almost three-quarters of total sperm defects (Table 1). Most of these defects occurred in the principal piece where they formed $51.7 \%$ of total defects, followed by the neck/midpiece region with $19.8 \%$ of total defects. A small incidence of defects $(0.6 \%)$ was apparent in the endpiece.

Anomalies of the neck/midpiece region manifested as midpiece bending (10.5\%), disjointed sperm (8.4\%) and abaxial tail implantation (0.9\%). Midpiece bending occurred at the proximal aspect of the midpiece (neck region), at the distal aspect of the midpiece (in the vicinity of the annulus) (Fig. 4b,c) or simultaneously at both localities (Fig. 4a). This resulted in the formation of a hairpin loop at the base of the sperm head, generally in association with residual cytoplasmic material (Fig. 4a,b). In some instances the bend formed a sharp reflex at the annulus resulting, in extreme cases, in shattering of the tail exposing elements of the axoneme (Fig. $4 \mathrm{~d})$.

Disjointed sperm, as described in the emu (Du Plessis and Soley, 2011b), were also a frequently observed defect of the midpiece ( $8.4 \%$ of total defects). It presented as the 


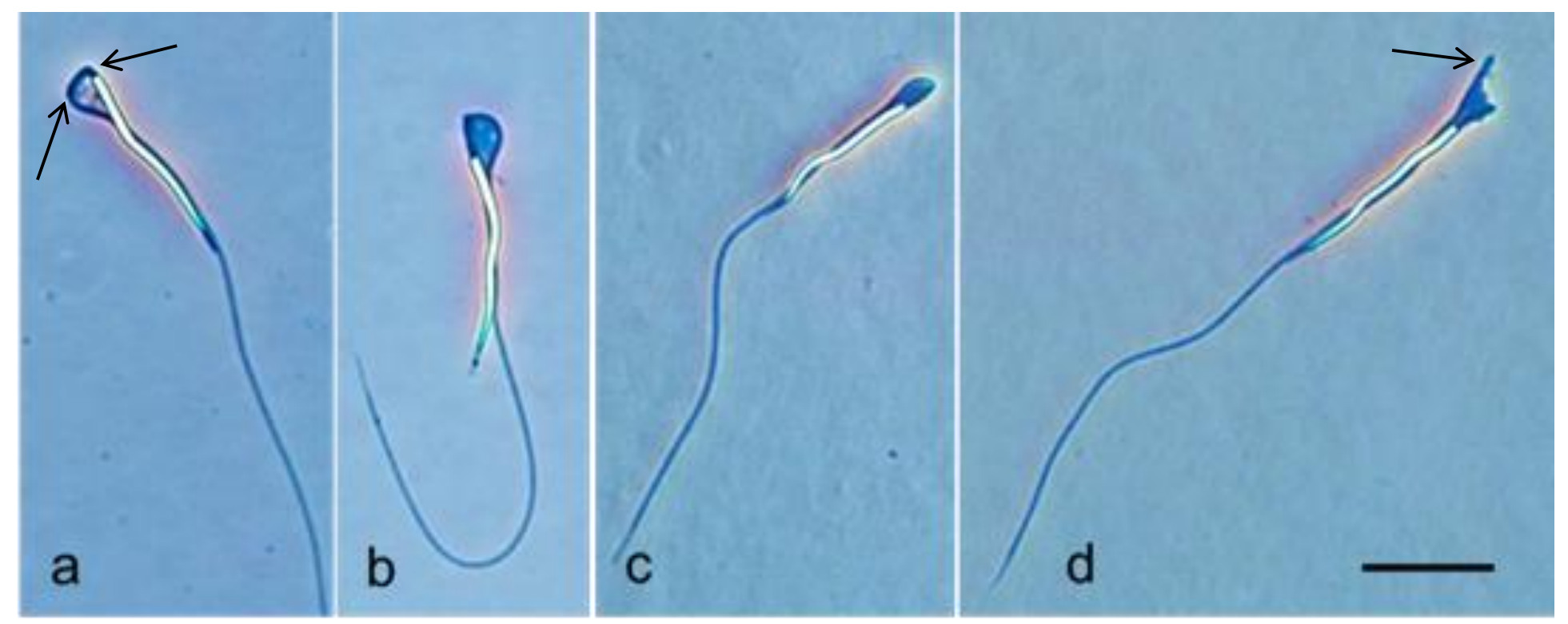

Fig. 4. Various forms of midpiece bending as observed in ostrich sperm. (a) Hairpin looping resulting from bending of the midpiece at the neck and annulus (arrows); (b), Gentle bending at the annulus (c) Sharp midpiece reflex; (d) Sharp midpiece reflex with shattering of the axoneme (arrow). Bar $=10 \mu \mathrm{m}$. 

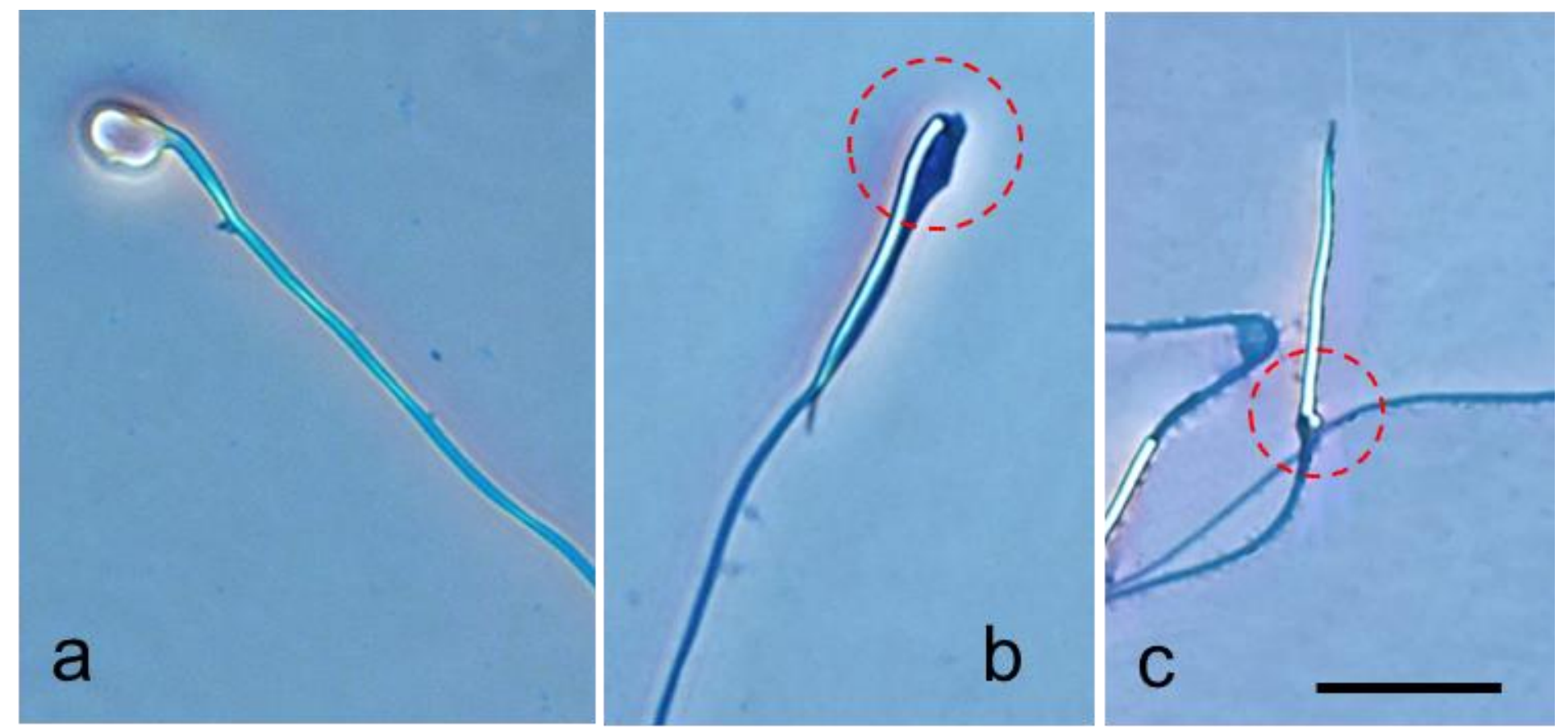

Fig. 5. (a) A large ostrich sperm with a deformed head. No obvious acrosome or midpiece is visible, and a visibly thicker principal piece is apparent. (b) Disjointed ostrich sperm (encircled); (c) Abaxial tail implantation (encircled). Bar $=10 \mu \mathrm{m}$. 
complete separation of the neck from the head-base at the connecting piece with a $180^{\circ}$ reflex of the tail, aligning the head and tail parallel to each other but within the confines of the plasmalemma (Fig. 5b). A third defect present in the midpiece region, although rarely noted, was abaxial tail implantation (Fig. 5c).

Bending of the principal piece was the most conspicuous defect (44.3\% of total defects) observed in this part of the tail. These bends were generally gentle, forming hairpin loops similar to those seen in the midpiece. Bends occurred at any position along the length of the principal piece, and commonly displayed trapped residual cytoplasmic material (Fig. 6). Sharp principal piece reflexes were also seen (Fig. 6f). Coiling of the principal piece close to the base of the head, reminiscent of the Dag defect in mammals (Barth and Oko, 1989), was also encountered (Fig. 7). In a few rare instances (only seen twice when examining the entire smear) the distal part of the principal piece was denuded due to the apparent loss of the surrounding fibrous sheath (Fig. 8a). This created the erroneous impression of an exceptionally long endpiece.

A small incidence $(0.1 \%)$ of multiple tails was observed, commonly in association with macrocephalic sperm. These tails presented as a single thick tail for most of the length of the principal piece the multiflagellate nature only being obvious towards the distal end of the 'compound' flagellum where the individual tails could be discerned (Fig. 9b). Coiling of the endpiece was occasionally noted and, when present, also involved the distal part of the principal piece (Fig. 8b).

Cytoplasmic droplets were commonly found on the smears, either as free droplets or attached to the sperm. Free droplets were occasionally associated with, but not attached to, the cells. Attached droplets, which appeared to form an integral part of the sperm, accounted for $17.5 \%$ of the total defects observed (Fig. 9a). The attached droplets were exclusively located at the base of the nucleus and proximal part of the midpiece. Residual 


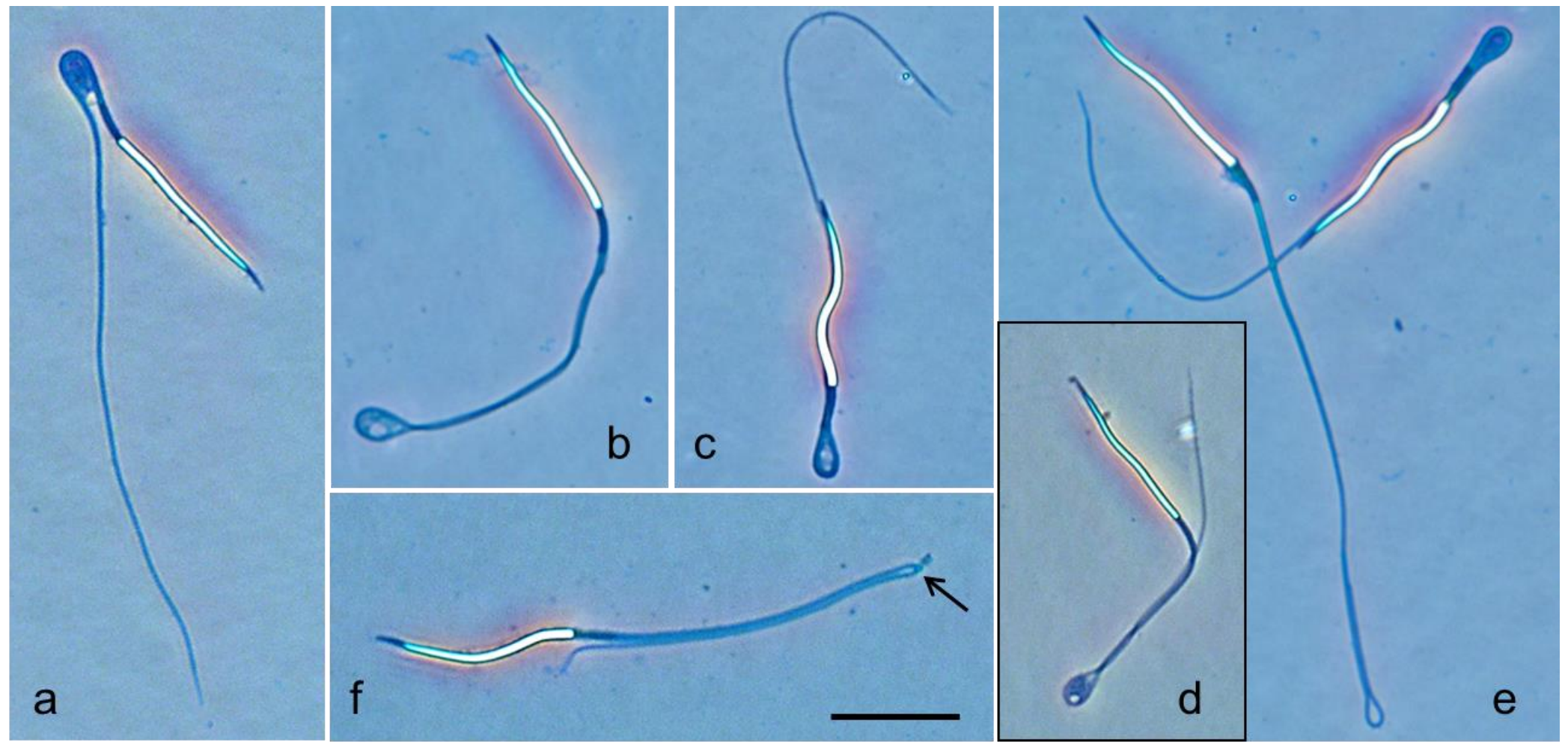

Fig. 6. (a-e) Gentle bending of the principal piece of ostrich sperm demonstrated at various positions along the length of the principal piece with cytoplasmic material present at the site of the bend (a,b,d,e). (f) Sharp reflex of the principal piece (arrow). Bar $=10 \mu \mathrm{m}$. 


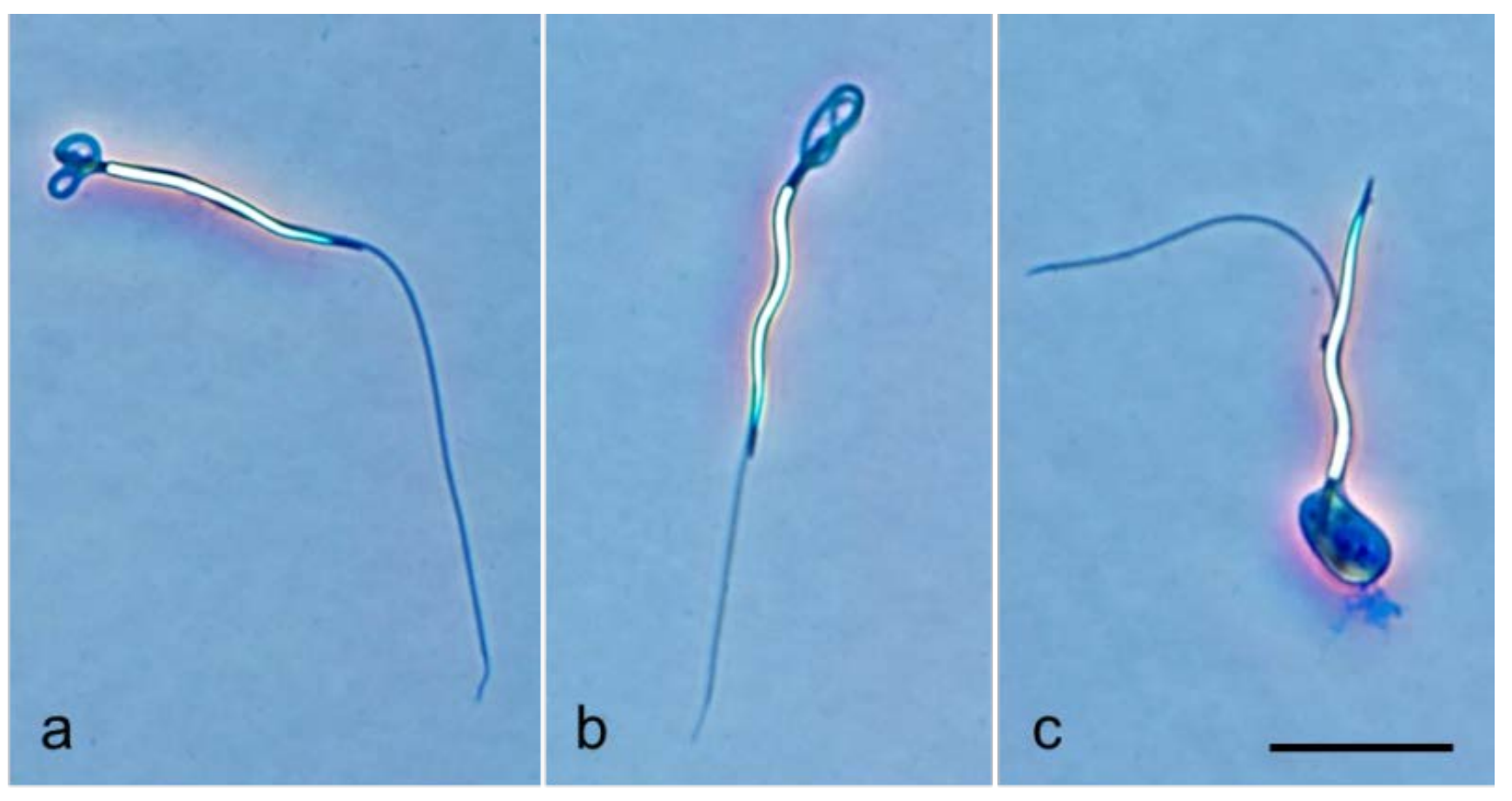

Fig. 7. Coiling of the principal piece of ostrich sperm reminiscent of the Dag defect in mammals. $(a, b)$ The coils occur at the base of the head but do not involve the midpiece. (c) The coils of the principal piece are trapped within a residual cytoplasmic mass. Bar $=10 \mu \mathrm{m}$. 


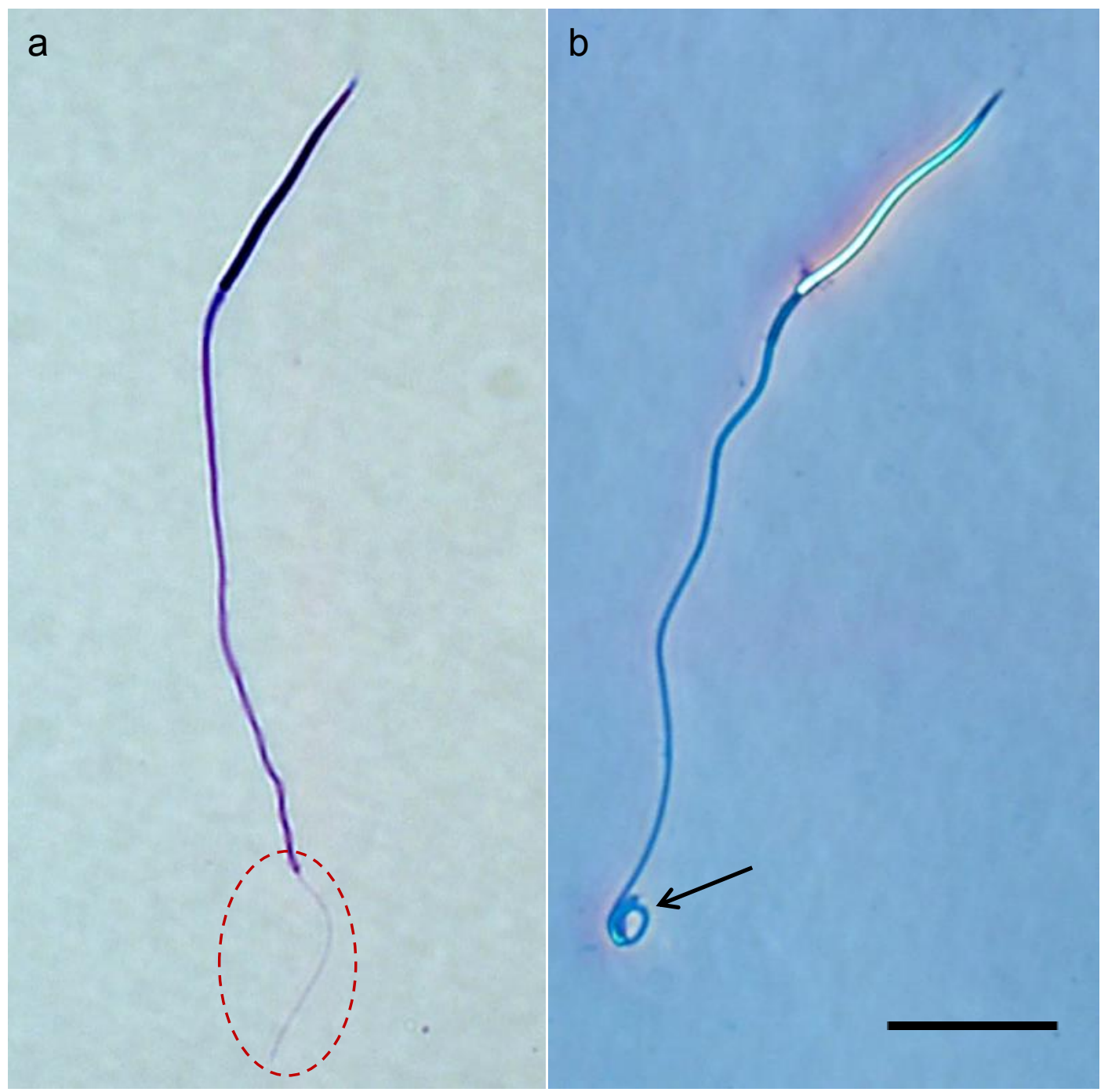

Fig. 8. Ostrich sperm displaying (a) a denuded distal principal piece showing absence of the fibrous sheath (encircled), as well as (b) coiling of the endpiece and part of the distal principal piece (arrow). Figure (a) bright field illumination; (b) phase contrast. Bar = $10 \mu \mathrm{m}$. 


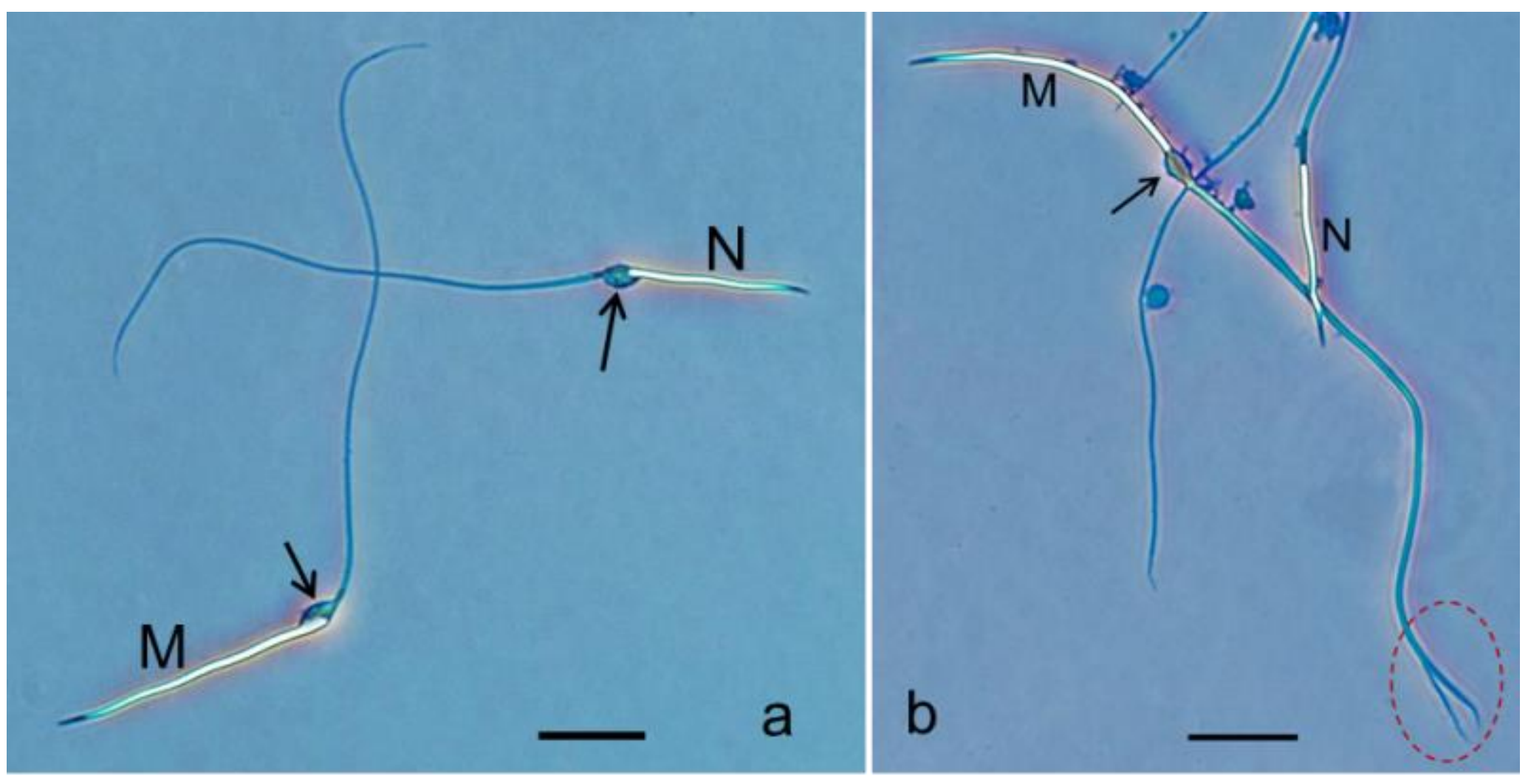

Fig. 9. (a) Cytoplasmic droplets (arrows) are shown overlapping the base of the nucleus and proximal region of the midpiece in these ostrich sperm cells. (b) A macrocephalic ostrich sperm demonstrating a thickened compound tail which separates into two individual tails in the distal region of the principal piece (encircled). A cytoplasmic droplet (arrow) covers the nuclear base. M, macrocephalic sperm; N, normal sized sperm. Bar $=10 \mu \mathrm{m}$. 


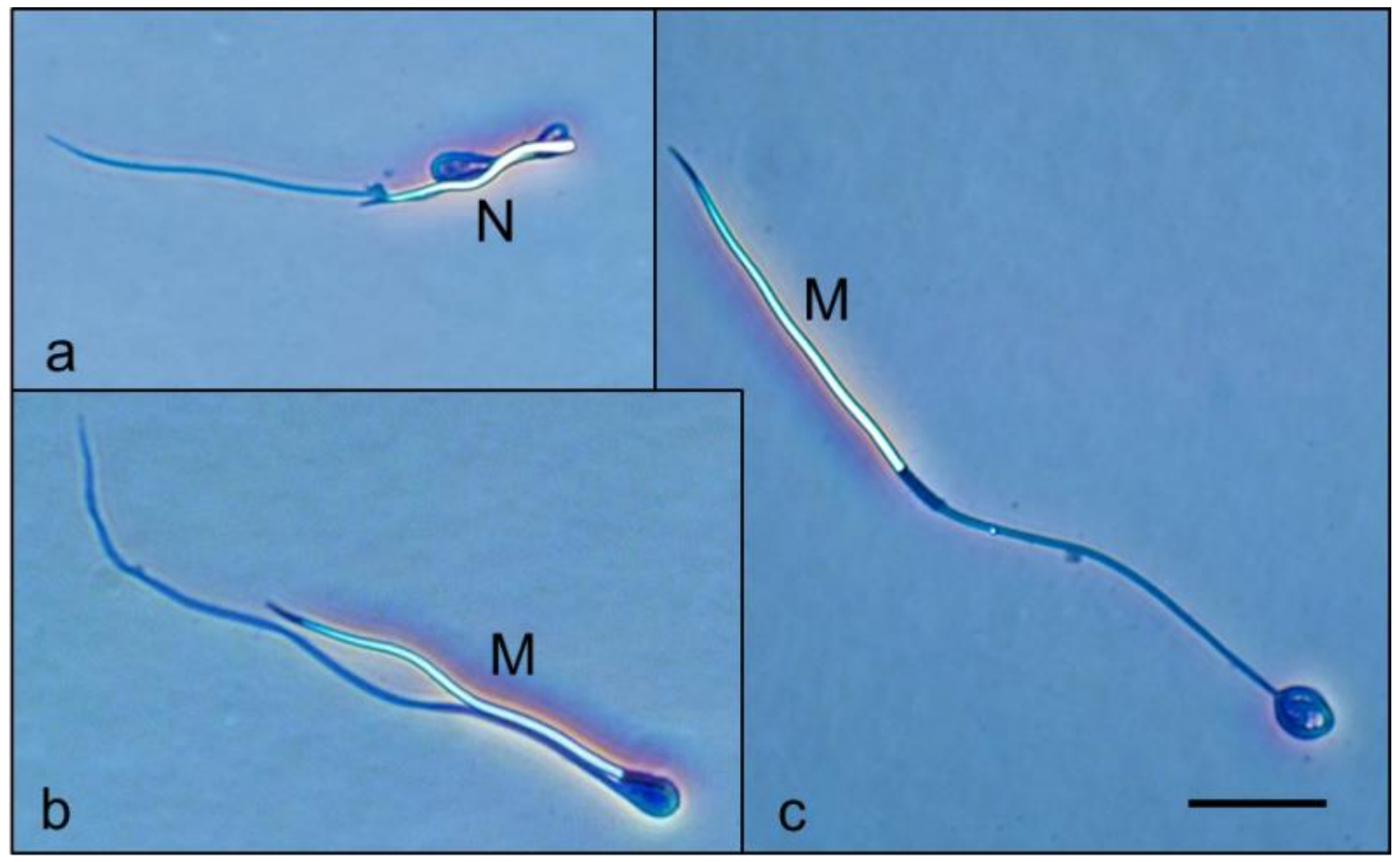

Fig. 10. Various combinations of multiple defects observed on ostrich semen smears. (a) Disjointed sperm with coiling of the principal piece; (b) Macrocephalic sperm with a gentle bend at the annulus; (c) Macrocephalic sperm displaying a coil involving the distal part of the tail. $\mathrm{N}$, normal sized sperm; $\mathrm{M}$, macrocephalic sperm. Bar $=10 \mu \mathrm{m}$. 
cytoplasmic material was often associated with other defects, especially bending of the head, midpiece or principal piece (Figs. 3, 4, 6).

Multiple defects were occasionally observed (1.2\% of total defects). This category was defined as sperm which displayed a combination of two or more of the above defects (Figs. $9 b, 10)$.

\section{Discussion}

\subsection{General incidence of sperm defects}

Although there have been a number of studies on semen characteristics in the ostrich (Bertschinger et al., 1992; Irons et al., 1996; Hemberger et al., 2001; Rozenboim et al., 2003; Soley and Els, 1993; Soley et al., 1996), very little information regarding the incidence of defective sperm in this species has been presented. Based on data from two birds, Rozenboim et al. (2003) reported an incidence of 5\% abnormal sperm whereas Hemberger et al. (1996) recorded a range of $5 \%$ to $26 \%$, with an average value of $17 \%$, in 56 birds. Bertschinger et al. (1992) reported a similar value of an average of $25 \%$ defective sperm in a study of 30 birds. Irons et al. (1996) evaluated ostrich sperm morphology on glutaraldehyde-fixed unstained smears using phase contrast microscopy and reported a value of $22 \%$ to $28 \%$ abnormal sperm. The current study, drawing on data from five ostriches, revealed a mean value of $17 \%$ (range $10.3-32.2 \%$ ) abnormal sperm, which is similar to that reported by Hemberger (1996). In another commercially important ratite, the emu, Du Plessis and Soley (2011a) and Du Plessis (2013) reported similar values (17\% and $13.6 \%$ ) for the emu on four and 15 birds, respectively. These results also agree in general with figures for domestic poultry, with similar values of $17 \%$ abnormal sperm being reported in the turkey (Alkan et al., 2002), 18\% in the pintail duck (Penfold et al., 2000) and $25 \%$ in the rock pigeon (Sontakke et al., 2004). When comparing the available 
data on ostrich semen it is interesting to note that the mean incidence of defective sperm displays a similar value irrespective of the collection method used, that is, digital manipulation (Bertschinger et al., 1992; Hemberger et al., 1996; Irons et al., 1996; Rozenboim et al., 2003) or use of an artificial cloaca (present study). No differences in the incidence and range of sperm defects were observed between the two older birds ( 7 and 8 years respectively) and the three younger birds ( 3 years old) used in the trial. However, ideally a larger sample size would provide more accurate information in this regard.

\subsection{Morphology and incidence of specific defects}

With the exception of the current study and data on the occurrence of cytoplasmic droplets (Soley and Els, 1993; Soley et al., 1996), there is no available information on the incidence of specific sperm defects in the ostrich. This situation is complicated by the lack of published information on sperm anomalies in this species and the resultant absence of a uniform system for their classification. However, a detailed description and classification of sperm defects has been presented for the emu (Du Plessis and Soley, 2011a) and provides a useful comparison for the results of the current study. When compared to data on the emu (Du Plessis and Soley, 2011a, b), it is clear that similarity exists in respect of the types of morphological defects observed in the two species. However, although the range of structural abnormalities is similar, a distinct difference is noted in the incidence of specific defects.

\subsubsection{Head defects}

Whereas head defects accounted for only $9.2 \%$ of total sperm defects in the ostrich, they formed the greatest proportion of defects in the emu (57.2\%) (Du Plessis and Soley, 2011a). Despite the striking difference in the relative proportion of this category between 
the two species, the range of head defects observed in ostrich sperm was similar to that described in the emu (Du Plessis and Soley, 2011a). A notable feature in both ratite species was the absence of acrosomal defects, although it is suggested that this category be included in the classification system for ratite sperm (Du Plessis and Soley, 2011a). Acrosome defects have been reported in other avian species. Ferdinand (1992) described a wide range of acrosome defects in the goose and Klimowicz et al. (2005), without providing any detail, mentioned the presence of acrosome defects in the pigeon. Maeda et al. (1986) reported swollen acrosomes in chickens, although this only occurred after the cells had been treated with cryo-protectants.

Macrocephalic sperm formed the largest component of head defects in the ostrich, the only other notable defect in this region being bent heads. Although macrocephalic heads also formed a large proportion of total head defects in the emu (18.3\%), bent heads, acephalic sperm and round heads collectively contributed to the greater proportion of head defects prevalent in the emu compared to the ostrich. Macrocephalic sperm are commonly found in small numbers in other avian species, such as the guinea fowl (Nwakalor et al., 1988), turkey (Lindsay et al., 1999), pigeon (Klimowicz et al., 2005) and quail (Chelmońska et al., 2008). High numbers of macrocephalic sperm (5-40\%) have been reported in the Houbara bustard without any apparent influence on fertility (Lindsay et al., 1999). Whether the large sperm with deformed heads of the ostrich represented another form of macrocephalic sperm could not be determined in the present study.

The occasional occurrence of microcephalic sperm in the ostrich was an interesting observation. In these cells the head was approximately half the length of normal sperm heads, but otherwise structurally normal. Microcephalic heads have not previously been reported in ratites and their presence appears to be rare in birds in general. This defect has 
been noted in the Northern pintail duck (Penfold et al., 2000) and goose (Ferdinand, 1992), but no incidence was reported in either study.

\subsubsection{Tail defects}

The most striking feature of this study was the high incidence of tail defects observed in the ostrich (72\%) compared to the emu (23\%) (Du Plessis, 2013; Du Plessis and Soley, 2011a). Most tail defects involved bending or coiling in all regions of the tail (including the endpiece) although this phenomenon was particularly obvious in the principal piece where it accounted for $52 \%$ of total defects. Tail bending has not been described in the emu (Du Plessis and Soley, 2011a) although it has been reported in a number of birds (Ferdinand, 1992; Nwakalor et al., 1988; Omeje and Marire, 1990; Penfold et al., 2000; Sontakke et al., 2004; Umapathy et al., 2005) with a variable incidence. Why tail bending should be so prevalent in the ostrich and relatively scarce in the emu, another commercially exploited ratite, remains enigmatic but may be related to the greater incidence of retained cytoplasmic droplets and residual cytoplasmic material associated with ostrich sperm (see below). To what extent the collection and preparation of semen from the distal deferent duct in the emu (Du Plessis and Soley, 2011a), as opposed to the processing of ejaculates collected in an artificial cloaca, might influence sperm structure, remains unknown. As previously reported in the emu (Du Plessis and Soley, 2011a), coiling of the principal piece in the vicinity of the head base of some ostrich sperm appeared characteristic of the "Dag" defect described in mammals (Barth and Oko, 1989) although this could not be confirmed.

Midpiece abnormalities appear to be a common defect of avian species, particularly defects described as 'bent midpiece' and 'deformed midpiece' (Chelmońska et al., 2008; Klimowicz et al,. 2005; Maeda et al., 1984; Nwakalor et al., 1988; Penfold et al., 2000; 
Siudzińska and Łukaszewicz, 2008; Umapathy et al., 2005). In the ostrich, midpiece defects were almost equally distributed between bending of the neck/midpiece and disjointed sperm. Whereas midpiece bending has not been described in the emu (Du Plessis and Soley, 2011a), this defect displays characteristic features in ostrich sperm where the bending occurs specifically at the neck or annulus or both regions simultaneously. The association of remnant cytoplasmic material with this particular form of tail bending and the location of the bend strongly suggests the involvement of the cytoplasmic droplet in the formation of the anomaly during spermiation. Disjointed sperm represent a unique neck/midpiece defect previously only identified in the emu (Du Plessis and Soley, 2011b). It is noteworthy that both ratite species display a similar incidence of this defect with $8.4 \%$ being recorded in the ostrich and $7.2 \%$ in the emu.

Bertschinger et al. (1992) described aplasia of the midpiece in their study on ostrich sperm morphology, although this phenomenon was not observed in the present study. However, denuded distal principal pieces were noted in a small number of ostrich sperm, a feature not observed in the emu or reported in other avian species. Whether this phenomenon is due to failure of the fibrous sheath to form in the more distal aspect of the principal piece, or that affected sperm simply display an abnormally long endpiece, remains a moot point.

\subsubsection{Cytoplasmic droplets}

Cytoplasmic droplets appear to be a persistent feature of ostrich semen and their presence has been reported by various authors (Bertschinger et al., 1992; Irons et al., 1996; Soley and Els, 1993; Soley et al., 1996). Soley et al. (1996) reported an incidence of up to $12 \%$ cells with retained cytoplasmic droplets, while the current study revealed a slightly greater percentage. Cytoplasmic droplets were also commonly observed in the emu, 
although at a lower incidence of $10.5 \%$ (Du Plessis, 2013). Retained droplets were mostly associated with the head-neck region. However, in some instances, particularly when bending of the midpiece or principal piece was involved, cytoplasmic material was commonly present at the bend. Whether the presence of this residual cytoplasmic material precipitates the bending, remains to be established. A large number of free-lying droplets, always of similar size, were prominent in the ostrich semen smears but notably absent from emu smears.

\subsubsection{Multiple defects}

Multiple defects were much less frequently observed in the current study on the ostrich (1.2\%) compared to the $11.5 \%$ multiple defects reported in the emu (Du Plessis, 2013). The literature is silent on multiple defects in birds although this is a category regularly seen in classification systems for mammalian sperm defects.

In conclusion, this study revealed a similar range of morphologically defective sperm in the ostrich as reported for the emu. However, the incidence of the defects differed markedly between the two species with head defects dominating in the emu and tail defects in the ostrich. To what extent the type of semen sample examined (from the ductus deferens of slaughter birds in the emu (Du Plessis, 2013; Du Plessis and Soley, 2011a) and ejaculates from live birds in the ostrich (present study)) may have influenced these results could not be determined. The results further indicate that a common classification system for ratite sperm anomalies is feasible. Such a system will be of practical value for the implementation of AI programs currently being developed for economically sensitive niche enterprises such as ostrich and emu farming. 


\section{Acknowledgments}

We thank the personnel of the Oudtshoorn Research Farm for their assistance. Partial funding for the project was provided by the Western Cape Agricultural Research Trust, the South African Ostrich Business Chamber and the National Research Foundation (NRF) of South Africa through their THRIP program.

\section{References}

Alkan, S., Baran, A., Özdaş, Ö.B., Evecen, M., 2002. Morphological defects in turkey semen. Turk. J. Vet. Anim. Sci. 26, 1087-1092.

Baccetti, B., Burrini, A.G., Falchetti, E., 1991. Spermatozoa and relationships in Palaeognath birds. Biol. Cell 71, $209-216$.

Barth, A.D. and Oko, R.J., 1989. Abnormal morphology of bovine spermatozoa. Iowa State University Press, Ames.

Berens von Rautenfeld, D., 1977. Mitteilungen zur künstlichen Besamung, Geschlechts und Altersbestimmung beim Strauß (Struthio camelus australis, Gurney). Der praktischeTierarzt. 5/77, 359-364.

Bertschinger, H.J., Burger, W.P., Soley, J.T., de Lange, J.H., 1992. Semen collection and evaluation of the male ostrich. Proc. Biennial Congr. South Afr. Vet. Assoc., Grahamstown, pp.154-158.

Bonato, M., Rybnik, P.K., Malecki, I.A., Cornwallis, C.K., Cloete, S.W.P., 2010. Between male variation in semen characteristics and preliminary results on the dilution of semen in the ostrich. S. Afr. J. Anim. Sci. 40, 438-441.

Bonato, M., Rybnik, P.K., Malecki, I.A., Cornwallis, C.K., Cloete, S.W.P., 2011. Twice daily collection yields greater semen output and does not affect male libido in the ostrich. Anim. Reprod. Sci. 123, 258-264. 
Bonato, M., Cornwallis, C.K., Malecki, I.A., Rybnik-Trzaskowska, P.K., Cloete, S.W.P., 2012. The effect of temperature and $\mathrm{pH}$ on the motility and viability of ostrich sperm. Anim. Reprod. Sci. 133, 123-128.

Chelmońska, B., Jerysz, A., Łukaszewicz, E., Kowalczyk, A., Malecki, I., 2008. Semen collection from Japanese Quail (Coturnix japonica) using a teaser female. Turk. J. Vet. Anim. Sci. 32, 19-24.

Ciereszko, A., Rybnik, P.K., Horbańczuk, J.O., Dietrich, G.J., Deas, A., Słowińska, M., Liszewska, E., I.A. Malecki, I.A., 2010. Biochemical characterization and sperm motility parameters of ostrich (Struthio camelus) semen. Anim. Reprod. Sci. 122, 222228.

Cloete, S.W.P., Engelbrecht, A., Olivier, J.J., Bunter, K.L., 2008. Deriving a preliminary breeding objective for commercial ostriches: an overview. Aust. J. Exp. Agr. 48, 12471256.

Du Plessis, L., 2013. The morphology and development of normal and abnormal spermatozoa in the emu, Dromaius novaehollandiae. PhD Thesis, University of Pretoria, Pretoria.

Du Plessis, L., Soley, J.T., 2011a. Incidence, structure and morphological classification of abnormal spermatozoa in the emu (Dromaius novaehollandiae). Theriogenology 75 , $589-601$.

Du Plessis, L., Soley, J.T., 2011b. Head-base bending and disjointed spermatozoa in the emu (Dromaius novaehollandiae): a morphological comparison of two closely related defects. Theriogenology 76, 1275-1283.

Du Plessis, L., Soley, J.T., 2014. Light microscopic features and morphometry of sperm in the emu (Dromaius novaehollandiae). Theriogenology 81, 203-209. 
Ferdinand, A., 1992. Licht- und elektronenmikroskopische Untersuchungen zur Morphologie von Ganterspermatozoen. PhD Thesis, University of Veterinary Medicine, Hannover.

Gee, F.G., Bertschinger, H., Donoghue, A.M., Blanco, J., Soley, J., 2004. Reproduction in nondomestic birds: Physiology, semen collection, artificial insemination and cryopreservation. Poult. Avian Biol. Rev. 15, 47-101.

Hemberger, Y., 1996. Spermatological examination and artificial insemination of ostriches. In: Deeming, D.C. (Ed.), Improving our understanding of ratites in a farming environment. Ratite Conference, Manchester, pp. p155-157.

Hemberger, M.Y., Hospes, R., Bostedt, H., 2001. Semen collection, examination and spermiogram in ostriches. Reprod. Domest. Anim. 36, 241-243.

Irons, P.C., Bertschinger,H.J., Soley, J.T., Burger, W.P., 1996. Semen collection and evaluation in the ostrich. In: Deeming, D.C. (Ed.), Improving our understanding of ratites in a farming environment. Ratite Conference, Manchester, pp. 157-159.

Jamieson, B.G.M., 2007. Avian spermatozoa: Structure and Phylogeny. In: Jamieson, B.G.M. (Ed.), Reproductive Biology and Phylogeny of Birds Part A. Science Publishers, Jersey, pp. 349-511.

Kawka, M., Horbańczuk, J.O., Sacharczuk, M., Zięba, G., Łukaszewicz, M., Jaszczak, K., Parada, R., 2007. Genetic Characteristics of the Ostrich Population Using Molecular Methods. Poultry Sci. 86, 277-281.

Klimowicz, M., Łukaszewicz, E., Dubiel, A., 2005. Effect of collection frequency on quantitative and qualitative characteristics of pigeon (Columba livia) semen. Brit. Poultry Sci. 46, 361-365.

Lambrechts, H., Swart, D., Cloete, S.W.P., Greyling, J.P.C., van Schalkwyk, S.J., 2004. The influence of stocking rate and male:female ratio on the production of breeding 
ostriches (Struthio camelus spp.) under commercial farming conditions. S. Afr. J. Anim. Sci. 34, 87-96.

Lindsay, C., Staines, H.J., McCormick, P., McCullum, C., Choulani, F., Wishart, G.J., 1999. Variability in the size of the nucleus in spermatozoa from Houbara bustards, Chlamydotis undulate undulate. J. Reprod. Fertil. 117, 307-313.

Maeda, T., Terada, T., Tsutsumi, Y., 1984. Comparative study of the effects of various cryoprotectants in preserving the morphology of frozen and thawed fowl spermatozoa. Brit. Poultry Sci. 25, 547-553.

Maeda, T., Terada, T., Tsutsumi, Y., 1986. Studies of the factors causing abnormal acrosomes and crooked-necks in fowl spermatozoa during freezing and thawing. Brit. Poultry Sci. 27, 695-702.

Malecki, I.A., Rybnik, P.K., Martin, G.B., 2008. Artificial insemination technology for ratites: a review. Aust. J. Exp. Agr. 48, 1284-1292.

Nwakalor, L.N., Okeke, G.C., Njoku, D.C., 1988. Semen characteristics of the guinea fowl, Numida meleagris meleagris. Theriogenology 29, 545-554.

Omeje, S.S.I., Marire, B.N., 1990. Evaluation of the semen characteristics of adult cocks of different genetic backgrounds. Theriogenology 34, 1111-1118.

Penfold, L.M., Wildt, D.E., Herzog, T.L., Lynch, W., Ware, L., Derrickson, S.E., Monfort, S.L., 2000. Seasonal patterns of LH, testosterone and semen quality in the Northern pintail duck (Anas acuta). Reprod. Fert. Develop. 12, 229-235.

Retzius, G., 1911. Spermien Ostafrikanischer Tiere. Biol. Unt., N.F. 16, 82-88. Taf XXV. Rozenboim, I., Navot, A., Snapir, N., Rosenstrauch, A., El Halawani, M.E., Gvaryahu, G., Degen, A., 2003. Method for collecting semen from the ostrich (Struthio camelus) and some of its quantitative and qualitative characteristics. Brit. Poultry Sci. 44, 607-11. 
Rybnik, P.K., Horbańczuk, J.O., Naranowicz, H., Łukaszewicz, E., Malecki, I.A., 2007. Semen collection in the ostrich (Struthio camelus) using a dummy or a teaser female. Brit. Poultry Sci. 48, 635-643.

Rybnik, P.K., Horbańczuk, J.O., Łukaszewicz, E., Malecki, I.A., 2012. The ostrich (Struthio camelus) ejaculate - effects of the method of collection, male age, month of the season, and daily frequency. Brit. Poultry Sci. 53, 134-140.

Siudzińska, A., Łukaszewicz, E., 2008. Effect of semen extenders and storage time on sperm morphology of four chicken breeds. J. Appl. Poultry Res. 17, 101-108.

Soley, J.T., 1992. A histological study of spermatogenesis in the ostrich (Struthio camelus). $\mathrm{PhD}$ Thesis, University of Pretoria, Pretoria.

Soley, J.T., 1993. Ultrastructure of ostrich (Struthio camelus) spermatozoa: I. Transmission electron microscopy. Onderstepoort J. Vet. Res. 60, 119-130.

Soley, J.T., Els, H.J., 1993. The ultrastructure of retained cytoplasmic droplets in ostrich spermatozoa. Proc. Electron Microsc. Soc. South. Afr. 23,58.

Soley, J.T., Groenewald, H.B., 1999. Reproduction. In: Deeming, D.C. (Ed.), The Ostrich: Biology, Production and Health. CABI Publishing, Oxon, pp. 129-158.

Soley, J.T., Roberts, D.C., 1994. Ultrastructure of ostrich (Struthio camelus) spermatozoa: II. Scanning electron microscopy. Onderstepoort J. Vet. Res. 61, 239-46.

Soley, J.T., Bertschinger, H.J., Roberts, D.C., de Lange, D.H., Burger, W.P., 1991. Correlation between semen quality and egg fertility in ostriches. Seminar on Ostrich Research, Oudtshoorn, pp. 45-47.

Soley, J.T., Bertschinger, H.J., Els, H.J., Burger, W.P., 1996. The morphology and incidence of retained cytoplasmic droplets in ostrich spermatozoa. In: Deeming, D.C. (Ed.), Improving our understanding of ratites in a farming environment. Ratite Conference, Manchester, pp. 16-18. 
Sontakke, S.D., Umapathy, G., Sivaram, V., Kholkute, S.D., Shivaji, S., 2004. Semen characteristics, cryopreservation, and successful artificial insemination in the Blue rock pigeon (Columba livia). Theriogenology 62, 139-153.

Umapathy, G., Sontakke, S., Reddy, A., Ahmed, S., Shivaji, S., 2005 Semen characteristics of the captive Indian white-backed vulture. Biol. Reprod. 73, 1039-1045. 